\title{
Acompañamiento de prácticas pedagógicas de formación inicial: un self-study a partir del rol como profesora guía
}

\author{
Romina Lattanzi ${ }^{1}$ (D) @ \\ Carlos Mario Vanegas Ortega ${ }^{2}$ \\ ${ }^{1}$ Universidad Central de Chile, ${ }^{2}$ Universidad de Santiago de Chile
}

Resumen. En el contexto de mi experiencia como profesora de Biología y Ciencias en la educación media, hace cinco años, y cuando aún era profesora principiante, de manera accidental me correspondió ser profesora guía de futuros profesores de Biología y Ciencias. Esto tensionó mi quehacer profesional puesto que confundía mi rol como profesora de aula con el de profesora guía de prácticas. Ante esta situación me propuse como objetivo de investigación caracterizar la transformación de mis propios procesos reflexivos a través de un self-study sobre el acompañamiento como profesora guía de prácticas pedagógicas de profesores de Biología y Ciencias en formación inicial. Los resultados muestran que se tensionó mi proceso identitario con el rol, permitiéndome diferenciar mi rol como docente de aula, y avanzar hacia la comprensión de una nueva identidad como formadora de profesores. Además, me permitió comprender mi propia práctica pedagógica desde una concepción más compleja, que posibilita la incorporación de las perspectivas de la tríada, problematizar la relación entre la teoría y las experiencias de aula, así como darme cuenta sobre el alto nivel de influencia que tienen las dimensiones personal/familiar y emocional en las formas de interacción con los estudiantes y con los profesores en formación.

Palabras clave: práctica pedagógica; profesor guía; reflexión docente; self-study.

Acompanhamento de estágios pedagógicos de formação inicial: um self-study a partir do papel de professora orientadora

Resumo.No contexto da minha experiência como professora de Biologia e Ciências no ensino médio, há 5 anos, quando ainda era professora principiante, casualmente me correspondeu ser orientadora de futuros professores de Biologia e Ciências. Isto gerou uma tensão profissional, já que confundia o meu papel de professora de sala de aula com o de orientadora pedagógica. Perante esta situação, defini como objetivo de pesquisa caracterizar a transformação dos meus próprios processos de reflexão através de um self-study sobre o acompanhamento como orientadora pedagógica de estágios de profesores de Biologia e Ciências em formação inicial. Os resultados mostram que a tensão entre o meu processo identitário e o papel de orientadora me permitiu diferenciar o meu papel de professora de sala de aula e avançar para a compreensão de uma nova identidade como formadora de professores. Além disso, me fez entender a minha própria prática pedagógica a partir de uma concepção mais complexa, possibilitando a incorporação das perspectivas da tríade, problematizar a relação entre a teoria e as experiências de sala de aula, além de perceber o alto nivel de influência que as dimensões pessoal/familiar e emocional nas formas de interação com os alunos e com os professores em formação têm.

Palavras-chave: prática pedagógica; orientador; reflexão docente; autoestudo; self-study.

Accompaniment of initial training pedagogical practices: A self-study based on the role as a guide teacher

Abstract. In the context of my experience as a high school teacher of Biology and Sciences, 5 years ago, and while I was a teacher-in-training, accidentally I became the guide for other future Biology and Sciences teachers. This created some tension in my professional life because it blurred the lines between my role as a classroom teacher and my role as a guide for other teachers-in-training. In this situation, I decided the goal of my research would be to characterize the transformation of my own self-reflective processes through self-study of the role of a mentor and teacher of pedagogical practices in Biology and Sciences. The results show a tension in my identity process with this new role, allowing me to differentiate my role as a classroom teacher, and move forward towards a new identity as a teacher trainer or guide. In addition, it allowed me to understand my own educational practices from a more complex point of view, which allows for the inclusion of triad perspectives, the problematization of theory and classroom experiences, as well as realizing the high impact and influence which personal/family and emotional relations have in the ways one interacts with students and teachers-in-training.

Keywords: pedagogical/educational practice; trainer/mentor; educational reflection; self-study. 


\section{Introducción}

Las prácticas pedagógicas de formación inicial son un punto de intersección entre la formación universitaria y el ejercicio profesional, es en esta instancia donde se produce el primer acercamiento del docente en formación con la realidad escolar, por tanto, tiene un carácter complejo porque tensiona las dimensiones personal y social del sujeto (Dewey, 1964; Imbernón, 2001; Latorre, 2004; Correa, 2011; Domingo \& Gómez, 2014). La práctica pedagógica tiene valores y compromisos asociados a la responsabilidad que implica la profesión docente, pero esta responsabilidad no solo es atribuible al profesor en formación (PF), sino también al profesor tutor (PT) y al profesor guía (PG). El tutor es quien orienta las prácticas desde la universidad, mientras que el profesor guía es quien lo hace desde establecimiento educativo escolar, y para algunos autores, es este último quien tiene la mayor influencia en el desempeño del profesor en formación (Correa, Malo \& Gervais, 2013), y por tanto, resulta imprescindible que el profesor guía comprenda y se apropie de su rol como formador de formadores.

La práctica pedagógica es una instancia para tener la oportunidad de reflexionar acerca de la labor como profesor en formación inicial, con la ventaja de poder aprender de experiencias e iniciativas exitosas o dificultosas, y es aquí donde el profesor guía cumple una importante labor, para lo cual debe sentirse preparado para instar a que se generen estos momentos de reflexión, es decir, ofrecer una perspectiva de la práctica alejada de la aplicación de teoría, sino orientada al sujeto, a sus pensamientos, opiniones y propósitos. Para que tanto el profesor en formación como el profesor guía alcancen este crecimiento personal y profesional, el profesor guía se convierte en un foco interesante de estudio, ya que es el responsable directo de dirigir y apoyar las acciones del profesor en formación.

En el contexto de mi experiencia como profesora de Biología y Ciencias en la educación media, hace 5 años, y cuando aún era profesora principiante, de manera accidental me correspondió ser profesora guía de futuros profesores de Biología y Ciencias. Esto tensionó mi quehacer profesional puesto que confundía mi rol como profesora de aula con el de profesora guía de prácticas. Ante ello, surgieron las siguientes preguntas: ¿Cuáles son las características que debe tener una profesora guía de prácticas pedagógicas? ¿En qué se asemejan y diferencian, el rol de profesora de aula escolar y el rol de profesora guía de prácticas pedagógicas de formación inicial? ¿Cuáles son las estrategias que me permiten optimizar el acompañamiento de las prácticas de los profesores en formación? ¿Cómo la reflexión sobre mi rol como profesora guía de prácticas impacta mi desarrollo profesional docente? Ante estas preguntas, me propuse como objetivo de investigación caracterizar 
la transformación de los propios procesos reflexivos a través de un self-study sobre el acompañamiento como profesora guía de prácticas pedagógicas de profesores de Biología y Ciencias en formación inicial.

\section{Metodología}

La investigación se realizó desde un paradigma cualitativo con enfoque de self-study (Olarte, 2016; Russell \& Mena, 2016; Russell, Fuentealba \& Hirmas, 2016). Se trata de interpretar críticamente el papel de mí misma como formadora de profesores, analizando las consecuencias de la toma de decisiones personales, así como las características y matices de los contextos particulares en los que se desarrolla el acompañamiento de la práctica pedagógica (Clarke \& Erickson, 2004).

\subsection{Procedimiento de recogida y análisis de datos}

Cualquier self-study suele abordarse desde diferentes perspectivas teóricas que obligan a la utilización de instrumentos diversos (Brewer \& Hunter, 2006). El acceso a múltiples conjuntos de datos en un self-study aumenta la posibilidad de recoger la riqueza de la realidad estudiada. Por ello, recurriré a mi primera experiencia como profesora guía de prácticas para analizar dos fuentes de datos diferentes: datos emergentes desde mi mirada personal y datos emergentes desde una perspectiva externa.

Hace 5 años fui asignada como profesora guía de prácticas de tres profesores de Biología y Ciencias que estaban en distintos niveles de formación. Esto implicó acompañar una profesora en tercer año de formación (PF1), un profesor en último año de formación (PF2) y un profesor en segundo año de formación (PF3). Desde la mirada personal, escribí 8 bitácoras con incidentes críticos sobre mi proceso de acompañamiento a los profesores en formación. De manera paralela, participé de una investigación en donde se investigó mi desempaño como profesora guía de práctica, por tanto, los resultados de dicha investigación me entrega la mirada externa que me permite complejizar este self-study.

La ventaja de tomar los datos de acontecimientos de 5 años atrás, es que puedo tomar distancia y mirar mis acciones y posicionamientos como mayor objetividad, lo que me permite ser más rigurosa con el self-study. Como se observa en la figura 1, el diseño metodológico responde a los objetivos de 
la investigación, a partir de dos fases: análisis y meta-análisis. A continuación, para cada fase se describen los instrumentos de producción de datos y los procedimientos de análisis de la información.

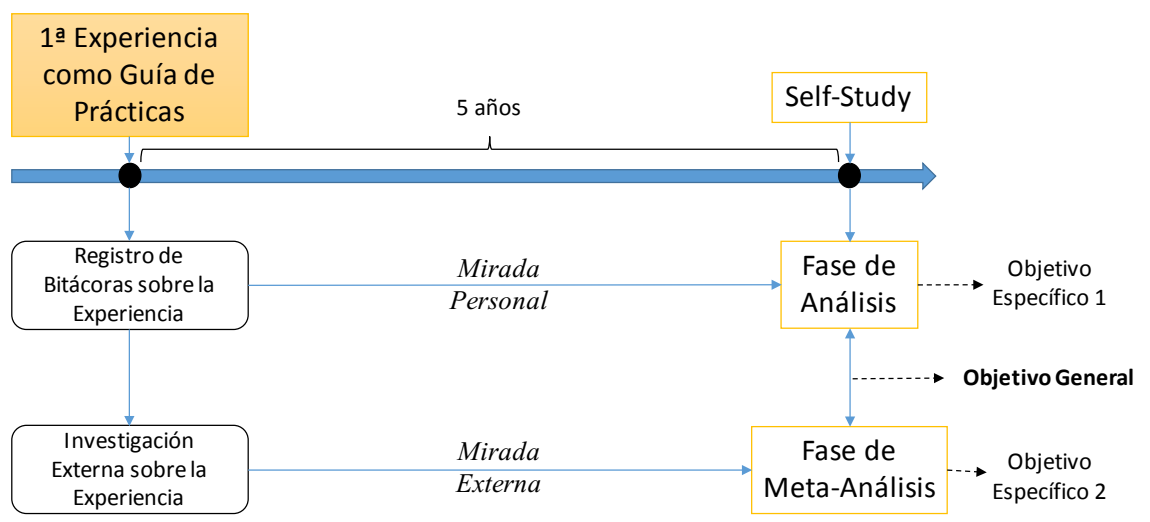

Figura 1. Diseño metodológico de la investigación. Fuente: elaboración propia.

Fase de análisis. Esta fase tuvo como objetivo específico identificar los propios procesos reflexivos que emergen del acompañamiento como profesora guía de prácticas pedagógicas de profesores de Biología y Ciencias en formación inicial. Para ello, se contó con el registro de 8 bitácoras de incidentes críticos donde escribí sobre mi proceso de acompañamiento a tres profesores de Biología y Ciencias en Formación. Cada bitácora aborda los elementos planteados en la tabla 1.

Tabla 1. Elementos considerados para el registro de bitácoras.

\begin{tabular}{lllll}
\hline Fecha & Descripción & ¿Qué sentimien- & ¿Por qué es & Si tuviese que enfren- \\
& general de la & tos o emociones & importante & tarme de nuevo a una \\
situación & me generó la & que registre & situación similar, ¿la \\
& situación? & este evento? & enfrentaría de la mis- \\
& & & ma manera?
\end{tabular}

Para el procesamiento de datos, se hizo análisis de contenido de cada bitácora a partir de las categorías definidas por Vanegas (2016, p. 132) para el estudio de procesos reflexivos de profesores de ciencias en contextos de prácticas pedagógicas. Para la reducción de información, se realizó la categorización de cada registro y se sintetizó los resultados en una matriz de resignificación.

Fase de meta-análisis. Esta fase tuvo como objetivo específico describir las convergencias y divergencias entre la perspectiva personal y la perspectiva de un investigador externo, cuando se analizan los procesos reflexivos sobre el propio acompañamiento como profesora guía de prácticas 
pedagógicas de profesores de Biología y Ciencias en formación inicial. Dado que, cuando tuve mi experiencia como profesora guía de prácticas, participé de una investigación en la que se estudió el desempeño de mi rol y los procesos de acompañamiento de los tres profesores en formación que me fueron asignados, para este self-study se contó, como punto de contraste y discusión reflexiva, con los datos, resultados y análisis del investigador.

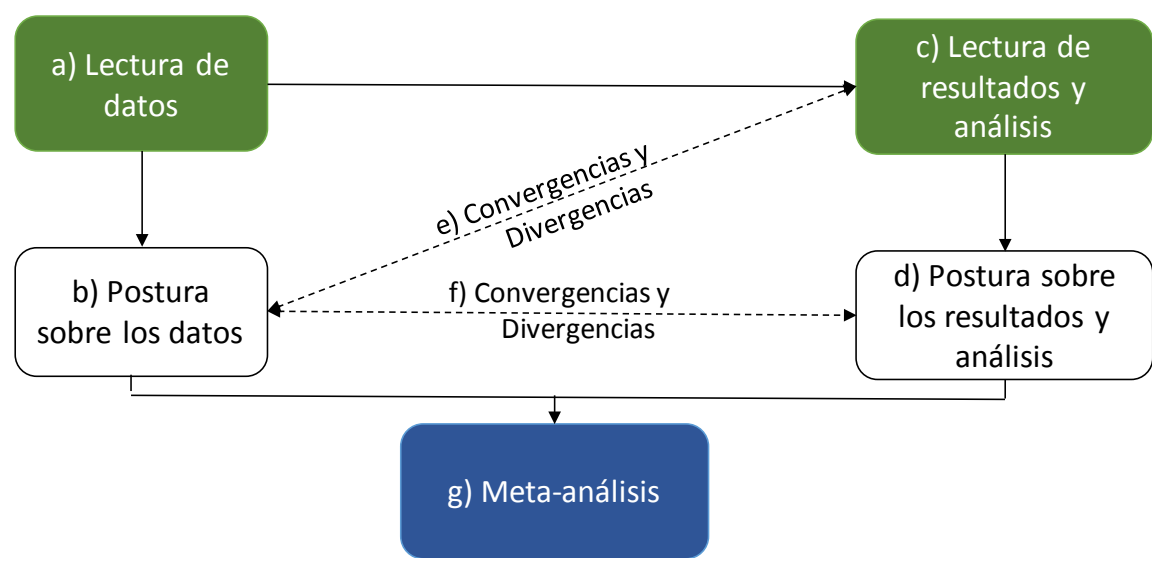

Figura 2. Procedimiento de análisis para la fase de Meta-Análisis. Fuente: elaboración propia.

Esta fase se ha denominado Meta-Análisis, puesto que implica el análisis (mío) del análisis que realizó el investigador (Olarte, 2016); además de las conversaciones presenciales con dicho investigador. De esa manera, se contrapone la perspectiva personal con la perspectiva externa. Como se muestra en la figura 2, para llegar a las conclusiones del meta-análisis se realizó sistemáticamente, para cada categoría, el siguiente procedimiento:

a) Lectura de los datos originales producidos por el investigador. Para la obtención de estos datos, el investigador me realizó dos entrevistas (una al inicio y otra al final) en las que abarcaba las categorías de análisis. Además, mi participación en la investigación implicó participar en dos momentos de trabajo con la tríada formativa de PF3: una mesa reflexiva tríadica y una entrevista grupal (Vanegas, 2016).

b) Establecimiento de una postura personal sobre el contenido de los datos originales.

c) Lectura de los resultados y análisis establecidos por el investigador.

d) Establecimiento de una postura personal sobre el contenido de los resultados y análisis establecidos por el investigador. 
e) Análisis de convergencias y divergencias entre b) y c).

f) Análisis de convergencias y divergencias entre b) y d).

g) Definición de conclusiones del Meta-Análisis.

Tanto para la fase de análisis como para la fase de meta-análisis, el rigor metodológico estuvo dado por procesos de triangulación entre investigadores (mi postura y la del investigador) y de fuentes (Vallejo y Finol de Franco, 2009). Para la fase de análisis, invité al investigador para que revisara la asignación de categorías a las bitácoras; en los casos que fue necesario, se hicieron ajustes. Para la fase de meta-análisis, además de la triangulación entre los datos y los análisis de los datos (procedimientos a y c de la figura 2), socialicé con el investigador mi postura personal sobre el contenido de los resultados y análisis establecidos por él, las discusiones me permitieron hacer modificaciones cuando había discrepancias en las interpretaciones o cuando se había omitido información.

\section{Resultados y análisis}

En coherencia con el diseño metodológico, primero presentaremos el análisis de las bitácoras, y posteriormente, realizaremos el meta-análisis de los datos asociados a la investigación sobre mi proceso de acompañamiento a la práctica de profesores en formación inicial de Biología y Ciencias.

\subsection{Análisis de bitácoras}

A continuación, se presenta el análisis las ocho bitácoras relacionadas con mi proceso de acompañamiento a la práctica de tres profesores en formación. En cada caso, se sintetizan los acontecimientos que detonan la reflexión, la mirada que le di hace cinco años, y finalmente, el análisis que hago hoy de la situación.

La primera bitácora surge cuando la PF1 llevó a los estudiantes de cuarto medio una guía tipo PSU (Prueba de Selección Universitaria) de biología para ser realizada en clase. Sin embargo, los estudiantes no se motivaron con la actividad propuesta por PF1 y plantearon que no la iban a realizar. Ante ello, le pedí a PF1 que la modificara ya que yo sentía que los estudiantes estaban poco motivados. Pero ella insistió en que se debía seguir la próxima semana con la misma guía, porque, según su criterio, los alumnos estaban muy débiles en biología. 
Esta bitácora corresponde a la categoría relación profesor en formación - profesor guía. En este caso, mi sentimiento era de frustración al no haber podido demostrar a PF1 que el instrumento de evaluación presentado a los estudiantes no estaba dando los resultados esperados. Hoy bajo una mirada más experimentada, considero que esta situación necesita otro tipo de análisis, en particular, esta actividad fue improvisada por PF1 (algo que no debió haber sucedido), confié en que PF1 me presentaría previamente el material y no fue así. Hoy, solicito a los PF que me entreguen los contenidos y actividades una semana antes. Esto facilita nuestra comunicación, además de darnos el tiempo para reflexionar y prever posibles situaciones que se podrían presentar. Además, este episodio se relaciona de manera directa con la categoría de Identidad como profesora guía, porque deja en evidencia la falta de apropiación de mi rol como profesora guía, ya que permití que PF1 improvisara y no generé argumentos e instancias de diálogo que le permitieran actuar de manera diferente.

La segunda bitácora se refiere al trabajo con PF2, a partir de una salida pedagógica a la que me acompañó, me sentí agradecida y tranquila al poder proponer ideas en conjunto, también poder realizarle correcciones y que él se las tomase a bien. Sentí que, con el transcurso del tiempo, PF2 ha ido modificando la actitud que tenía en un principio de querer resistirse a cambiar, a que yo le hiciera recomendaciones, le corrigiera o le diera ideas nuevas. Me llama la atención el hecho que el factor edad haya sido significativo para mí. Consideraba que, por tener edades similares, PF2 no me respetaría o no me consideraría como una guía. Esta situación instala un primer proceso de identificación con el rol de profesora guía, ya que no me sentía con la autoridad para formar a futuros profesores debido a mi poca experiencia en el rol y la poca confianza que se había desarrollado con PF2. Esta aprehensión pudo deberse a que, coherente con lo que plantean Gervais (2007) y Correa (2011), yo consideraba que ser profesora guía era una responsabilidad que tenía consecuencias inmediatas sobre la formación y desarrollo profesional del futuro profesor.

La tercera bitácora se relaciona nuevamente con PF1, se presentó un conflicto de aula debido a que PF1 descuidó una responsabilidad que le había encomendado. Esto me hizo sentir molesta, tener la sensación de que PF1 no estaba aportando al desarrollo de la clase y, además, actué precipitadamente proyectando mi molestia con los estudiantes. Lo complejo es que la situación no la dialogué con PF1, y creí que la manera de resolverla era siendo más autoritaria con ella.

Cuando un docente comparte su reflexión con sus pares se convierte en un estimulador de la reflexión de otros (Correa et al., 2013). La clave para poder superar este tipo de situaciones y que no se volvieran a repetir en el 
futuro, era haberlo analizado en ese instante con la PF1, ya que cuando el docente sociabiliza este proceso reflexivo, por medio de la verbalización, se habla de un proceso mancomunado que permite generar los cambios que en este caso yo quería que ella hiciera.

La cuarta Bitácora relata lo sucedido con PF2, quien realizó una actividad práctica de la unidad temática de los sentidos. Cuando me planteó lo que quería hacer, no me pareció interesante y pensé que los estudiantes iban a participar de mala manera, pero finalmente me llevé una grata sorpresa porque los jóvenes trabajaron muy bien y se notaban muy entusiasmados.

Esta situación es el ejemplo claro de que la formas de trabajar en el aula y las relaciones entre docentes pueden ser potenciadas al dar un rol más activo y confiar en las iniciativas de los PF (Montecinos \& Walker, 2010). En este caso hubo efecto sobre mis procesos reflexivos relacionados con la categoría de didáctica y ciencia; el profesor en formación llegó a mejorar mi ejercicio profesional, aportando conocimiento didáctico actualizado y pertinente para las características de los estudiantes.

La quinta bitácora corresponde a lo ocurrido con PF3, cuando le solicité a este que me ayudara a realizar una actividad en el laboratorio de computación encargándose de los estudiantes de las últimas filas. PF3 se dedicó a hacer otras cosas y no lo solicitado. El sentimiento que provocó en mí fue de cansancio y un poco de molestia debido a la falta de iniciativa del PF. En este caso, debido a mi falta de experiencia, le encargué al profesor en formación una actividad poco desafiante, ya que solo debía estar al cuidado de los estudiantes. Tal vez el haberle solicitado que los ayudara a realizar la actividad hubiese resultado mucho más motivador para él.

Este acontecimiento es un ejemplo de la categoría Identidad como Profesor Guía, ya que logré identificar que soy la responsable directa de generar en el PF, instancias de desarrollo profesional, encomendando tareas desafiantes y no limitando al PF a observar o ejercitar formulaciones teóricas, restringiendo los aportes que estos pueden hacer a la formación inicial docente.

La sexta bitácora tiene relación con el PF2, este fue de gran ayuda durante una salida pedagógica apoyándome con los estudiantes y con los trámites correspondientes a toda la salida, los sentimientos fueron de gratitud y confianza, ya que todo lo solicitado al PF fue realizado a tiempo y con profesionalismo. 
Esta situación está en directa correspondencia con la categoría Relación Profesor guía - Profesor en formación, ya que la confianza profesional entregada al PF era producto de la cercana relación laboral que tenía con él, debido al extenso tiempo que compartíamos y a los momentos para reflexionar acerca de nuestras experiencias. Este tipo de conocimiento se produce cuando hay relaciones interpersonales en un contexto común durante periodos prolongados de convivencia (MINEDUC, 2005)

La séptima bitácora se genera cuando me corresponde evaluar la práctica profesional de PF3, esta fue una instancia de reflexión importante para ambos, en donde pudimos socializar y discutir los acontecimientos más relevantes vividos durante su práctica. Me sentí cómoda durante la conversación, ya que ambos fuimos capaces de expresar con respeto, los sentimientos que se generaron durante el desarrollo de esta práctica profesional. También evidencié que habíamos tenido muy pocos momentos para reflexionar y realizar sugerencias a su labor como PF.

Lo expresado en el párrafo anterior, refleja las pocas instancias que los PG generamos para reflexionar en conjunto con el profesor en formación. Las actividades diarias muchas veces no permiten reservar momentos para contrastar experiencias pasadas y actuales, pero esto nunca debiese ser un impedimento para dialogar y llegar a acuerdos. Esta situación tiene que ver con la categoría de Identidad como Profesora Guía, ya que permitió darme cuenta de mi responsabilidad para generar momentos de reflexión que hubiesen ayudado a resolver los conflictos.

La octava bitácora hace referencia a una mesa reflexiva en la cual participamos PF3-PT-PG, un momento grato donde pudimos intercambiar opiniones, conocer los pensamientos y también saber cómo actúa o actuaría cada uno frente a determinadas situaciones. En la mayoría de las cosas llegamos a acuerdos, aunque existieron situaciones donde las opiniones eran bastante diferentes.

Esta forma de trabajo conjunto se relaciona con la categoría Relaciones Tríadicas (RT), la cual permite llevar a cabo procesos reflexivos grupales, abordando una dimensión social, porque es una actividad en la que pueden participar todos los implicados en el proceso formativo (Domingo \& Gómez, 2014). Este proceso cognitivo y afectivo de la relación tríadica permite también analizar situaciones que involucran a los tres actores, solucionar pugnas y llegar a acuerdos que en la relación diádica no serían posibles (Vanegas, 2016). 


\subsection{Meta-análisis}

Para el meta-análisis, abordaré en cada categoría los focos centrales de los resultados del investigador, y al mismo tiempo, plantearé las convergencias y divergencias que aparecen desde mi forma de ver los datos o desde mi forma de ver los análisis del investigador. Primero abordaré las categorías relacionadas con la identidad (idenficación, identización, identidad como profesora guía), luego las de contexto (contexto inmediato, sistema educativo, sociocultural, enseñanza), posteriormente las de las concepciones (práctica, reflexión, didáctica y ciencia), y finalmente las de la tríada (relaciones tríadicas, relación profesor en formación - profesor guía, relación profesor tutor - profesor guía).

\section{Identificación}

El investigador planteó que, según mi perspectiva, las características principales de los profesores son el contacto profundo que se tiene con los estudiantes y la desmotivación por la profesión, sin embargo, al remirar estos datos, realicé el cuestionamiento por la permanencia en la profesión, que puede estar influenciado por distintas variables; sin embargo, este cuestionamiento no está planteado desde una perspectiva negativa sino en el tipo de cuestionamiento que, según Schön (1987) y Dewey (1933), moviliza al profesorado a generar cambios en sus prácticas.

Con relación al contacto con los estudiantes, este aspecto cobra mayor importancia en la actualidad, ya que considero que el cambio que se puede evidenciar en las aulas de clases no es mínimo, por el contario, es un gran cambio el que puede generar el profesorado a partir de su rol en la escuela. Un aspecto a destacar es que la mirada que tengo ahora con respecto a lo que identifica al profesorado, es cómo en realidad soy consciente de que la labor que realiza un profesor puede estar mediada por dos roles que son distintos: la función como profesor y la función como profesor guía. Aunque esta última no es una dimensión de análisis para esta categoría, está influyendo desde la perspectiva como me posicionó para enfrentar la identidad de profesora.

\section{Identización}

La identización "es un proceso de individualización donde el sujeto decide qué elementos del grupo le son propios, cuáles adopta y cuáles no, qué lo hace similar al grupo y en qué se diferencia" (Vanegas, 2016, p. 64). En ese sentido, los elementos personales que logro destacar y que me pudiesen diferenciar dentro del colectivo profesional es que tanto en los análisis del investigador como en el meta-análisis que estoy realizando, pongo énfasis en los sentimientos que me genera el ver un avance en los aprendizajes de mis estudiantes y no solo en lo cognitivo sino también en lo afectivo, esto se 
refleja en las altas expectativas que tengo hoy con respecto a lo que pueden hacer mis estudiantes. Logro reflexionar a través de los cambios que se realizan en los jóvenes. La diferencia principal es que hoy hablo desde la experiencia, esta me permite ver la evolución que van teniendo los jóvenes y los logros alcanzados; en cambio, antes destacaba la importancia de trabajar los objetivos transversales del currículo, sin importarme si ello en realidad tenía alguna implicancia en el logro de sus metas personales.

\section{Identidad como profesora guía}

Según el investigador, aspectos relacionados con los 'procesos afectivos' tienen un gran valor para mí a la hora de guiar una práctica ; la mirada de hoy ratifica esa postura, ya que todavía sigue siendo importante el preocuparme y ocuparme de que el profesor en formación a mi cargo se sienta a gusto, valorado y que además sea él el protagonista y no la profesora guía (como sentí que había sido en mi práctica profesional).

La comprensión de mí misma evolucionó a algo más cercano a una profesora guía que a una profesora de aula, esto se debe a mi mayor experiencia como docente escolar, por lo que logro identificar las diferencias con mayor facilidad. Sigo destacando la importancia de los aprendizajes de saberes y recursos específicos del trabajo como profesor escolar, ya que no solo se evalúa a un profesor por sus buenas clases sino también por cumplir con tareas administrativas propias de un docente, por lo que me sigo preocupando de inculcar estos aprendizajes. Tanto en los datos del investigador como en sus análisis, logro identificar las diferencias entre ser profesora y ser profesora guía de prácticas pedagógicas de formación inicial, lo cual instala un proceso de identificación con el rol. Ser profesora guía es una gran responsabilidad para mí porque tiene consecuencias inmediatas sobre la formación y desarrollo profesional del futuro profesor (Correa, 2011; Vanegas, 2016), no así en mi rol como profesora de ciencias.

En la actualidad sigue existiendo una diferenciación de roles, pero hoy destaco el hecho de dejar de tener esa postura de 'modelo perfecto' en donde el profesor en formación solo rescate de mi práctica como docente lo positivo, sino que pueda más bien seleccionar lo más significativo de cada práctica y profesor guía y así crear su propio perfil como futuro docente. Esto me permite hoy relajarme más en el rol de profesora guía, ya que no es una camisa de fuerza en la que se tiene que ser perfecta. Ahora entendiendo mi labor como un espacio de aprendizaje para mí y un apoyo para los procesos reflexivos del profesor en formación.

\section{Contexto inmediato}

El investigador da cuenta que lo más relevante en ese momento para mí, eran las condiciones laborales en la que nos enfrentábamos los profesores, el contexto material me impedía sentirme a gusto desarrollando 
mi trabajo como profesora guía, ya que no contaba con todos los recursos materiales para realizar clases más diversas. Actualmente, por el contario, es un desafío el constantemente buscar nuevas estrategias para enseñar ciencias y así mostrarles a los profesores en formación, que es posible obtener resultados satisfactorios con condiciones que no son las ideales. Concuerdo también con lo planteado por el investigador, el cual menciona que el contexto dejó de ser un obstáculo, ya no busco igualar mis condiciones culturales con la de los estudiantes, sino que estoy realizando un proceso que me permite comprender y valorar las diferencias.

\section{Enseñanza}

Según el investigador, la reflexión inicial sobre el éxito de una clase fue determinado principalmente por la relación profesor - estudiante, la cual considero nunca debe ser de amistad, lo que no niega el surgimiento de lazos afectivos y sentimientos perdurables en el tiempo. El investigador destaca la importancia que tiene para mí el manejo de los contenidos y el conocimiento de la disciplina que enseño, el cual fortalece las interacciones con el estudiantado. Concuerdo plenamente con los resultados del estudio, ya que la dimensión de enseñanza está en directa relación con la dimensión de identización, porque involucro experiencias personales para determinar el tipo de relación que debo tener con los estudiantes y con los profesores en formación.

\section{Práctica}

Cuando comparé los datos con los resultados del investigador, no evidencié incoherencias con la concepción del proceso de práctica pedagógica que tengo hoy. Sin embargo, en el momento de escribir este meta-análisis me di cuenta que ahora tengo una comprensión de la práctica que es más compleja porque involucra la reflexión sobre las experiencias, el contexto, los otros (la tríada), y lo más relevante, los elementos emocionales derivados de las situaciones personales que modificaron mi identización (lo personal/ familiar influyendo en mi forma de ser y de ver la profesión docente). Tal vez lo más significativo es cuando analicé qué es para mí la práctica, en un comienzo lo consideré un espacio que desafía por cambios en la educación, hoy complementaría que es un punto de encuentro entre lo aprendido en la universidad y la resignificación de las experiencias de la labor docente. Inicialmente tenía una mirada más romántica de lo que es la pedagogía, en donde el cambio en la educación es lo que me movilizaba, hoy hay una mirada menos idealista basada en las experiencias y las condiciones de los contextos educativos.

En esta dimensión también aparecen nuevas ideas que me llaman la atención, por ejemplo, el hecho que la práctica esté condicionada por el estado de ánimo, esto se debe a que durante los últimos años, producto de 
una situación personal, pude vivenciar que las emociones o el momento en el cual nos encontremos es clave para condicionar la percepción que tenemos con respecto a la práctica. Antes no consideraba eso como un aspecto importante, creía que se podía separar lo personal de lo laboral, pero no es así, por lo tanto hoy me preocupo de saber cómo se encuentran mis estudiantes y el profesor en formación, incluso para considerarlo dentro de mi evaluación. En coherencia con la Comisión sobre Formación Inicial Docente (MINEDUC, 2005), la producción de conocimiento práctico depende de las relaciones interpersonales, por eso hoy quiero tener una relación que me permita transmitir no solo conocimiento práctico, sino también, formativo desde lo emocional. El investigador destaca de mi entrevista, la reflexión y la consideración de lo grupal como elementos que incluyo para posibilitar el desarrollo profesional docente, debiéndose esto a las dinámicas grupales de la investigación.

\section{Reflexión}

Dewey (1933) concebía la reflexión como un proceso sistemático y riguroso del cual se pueden construir significados y conclusiones. En mi caso, inicialmente considero la reflexión como un proceso cognitivo que mejora las prácticas pedagógicas, la cual imagino desde una forma individual y que se realiza de una manera más formal ya que ocurre en el establecimiento educacional. Según el investigador, la experiencia como profesora y formadora de profesores me ha permitido resignificar el concepto de reflexión al que le he incorporado nuevos elementos, como la reflexión en otros espacios diferentes al trabajo. Según Black y Plowright (2010) una de las dimensiones de la reflexión es el aprendizaje de tipo informal el cual considera actividades fuera del aprendizaje formal como actividades extra programáticas y lecturas recomendadas.

Otro elemento es que actualmente considero a otros individuos dentro de mi proceso reflexivo, es decir, la reflexión grupal toma un significado importante; como señalan Domingo \& Gómez (2014), cuando el docente internaliza el proceso reflexivo se habla de un proceso individual; cuando el docente sociabiliza ese proceso reflexivo, por medio de la verbalización con sus pares, se habla de un proceso grupal. Este cambio de paradigma sobre lo que es reflexión se debe principalmente al trabajo realizado durante las tríadas, ya que evidencié un aprendizaje comunitario y no individual.

\section{Didáctica y ciencia}

En un comienzo de la investigación, la didáctica y ciencia tenían un significado muy distinto al que tengo hoy, esto se debe a que me veo obligada a mantenerme actualizada constantemente a cerca de la didáctica de la ciencia, y a crear secuencias de actividades con el objetivo de enseñar ciencia para cada nivel y contexto sociocultural en donde me encuentre desarrollando mi trabajo. Según el análisis del investigador, consideraba el manejo del conoci- 
miento científico como una herramienta que me daba cierta autoridad frente al profesor en formación, es decir separaba el concepto de didáctica y de ciencia. En la actualidad ambos están fusionados, no funcionan por separado. Antes pensaba que el conocimiento de la disciplina era mi principal herramienta para apoyar el profesor en formación, hoy veo que este conocimiento tiene que ser complementado con un discurso pedagógico articulado al contexto educativo y, con conocimientos didácticos que permitan promover los aprendizajes científicos de todos los estudiantes. Ciencia, pedagogía y didáctica son tres focos de reflexión y trabajo con los profesores en formación.

\section{Relaciones tríadicas}

Tanto al inicio de la investigación como ahora, destaco la importancia del trabajo y la reflexión de y sobre la relación tríadica (profesor guía - profesor tutor - profesor en formación). Concuerdo con el investigador en que las mesas reflexivas tríadicas me sirvieron para ser consciente de la importancia del trabajo en tríada. Si me hubieran preguntado antes no hubiese considerado al profesor tutor como un actor significativo dentro de la práctica del profesor en formación, actualmente, comprendo que el profesor tutor juega un rol relevante ya que me permite tener una visión más acabada sobre el profesor en formación y sobre los objetivos del proceso de la práctica pedagógica.

Otro aspecto a destacar es el cambio en mi opinión respecto a la función que tiene el Profesor en Formación. La imagen de él ha evolucionado de un futuro profesor que ayuda a corregir evaluaciones y prepara material, a uno que no solo se prepara en los saberes teóricos sino también prácticos (Correa, 2011), que son orientados por mí durante la instancia de práctica pedagógica. Cabe destacar que, en esta dimensión, el análisis debe ser con respecto a los tres actores de la tríada, pero se evidencia que todavía me cuesta visualizar a los tres interactuando en conjunto, esto se debe a que aún no tengo instalado como práctica habitual el trabajo mancomunado con el profesor tutor; siempre que nos reunimos es en dupla, a menos que sea para la evaluación final del PF.

\section{Relación PF-PG}

Para Correa (2011), el profesor guía es quien articula los conocimientos prácticos y teóricos dentro del proceso de práctica del profesor en formación. En mi caso, destaco los aprendizajes y sentimientos que genera en mí esta relación diádica, lo que está en directa correspondencia con la categoría de identización; es decir, esta relación con el profesor en formación influye en mi experiencia, emociones, valores, desafíos, entre otros aspectos que me diferencian del resto de mis pares. Hoy puedo decir que además de todo lo que he aprendido de los profesores en formación, y evitando siempre un trato jerárquico, también considero mi aporte a su formación. Como lo 
explícita Correa (2011), propiciando el aprendizaje experiencial por medio de la reflexión, mi rol de PG implica el cuestionamiento y retroalimentación durante todo el proceso.

\section{Relación PT-PG}

Durante el estudio del investigador, la relación diádica con el profesor tutor no ocurría, por tanto, nuestra interacción fue mínima, esto traía consigo consecuencias importantes para los procesos de los profesores en formación (Figueira \& Rivas, 2011; Frick, Carl \& Beets, 2010); pero lo interesante según el investigador, y en lo que concuerdo, es que la entrevista grupal y la mesa reflexiva tríadica nos dio la posibilidad de interactuar en otras condiciones. Esto sirvió para fortalecer la relación con el profesor en formación, ya que tenía una perspectiva más clara con respecto a las actividades que debía o no realizar para cumplir con los requisitos mínimos esperados por la universidad y, las expectativas formativas que se requerían para aprobar la práctica pedagógica. Hoy al mirar y recordar la poca comunicación con el profesor tutor, no sólo pienso en las consecuencias que ello tuvo en el profesor en formación, sino en mi desarrollo profesional, y en particular, en tener perspectivas más amplias sobre las responsabilidades que implica ser profesora guía.

\section{Conclusiones}

En cumplimiento del primer objetivo específico del self-study, el análisis de las bitácoras permite concluir que, durante mi primera experiencia como profesora guía de prácticas pedagógicas de profesores de Biología y Ciencias en formación inicial, emergen procesos reflexivos sobre mi identidad como profesora guía, la relación diádica profesor en formación - profesor guía, y las concepciones de reflexión y didáctica de las ciencias.

El principal logro de la primera fase de la investigación fue darme cuenta cómo la primera experiencia como profesora guía tensionó mi proceso identitario con el rol, permitiéndome diferenciar mi rol como docente de aula, y avanzar hacia la comprensión de una nueva identidad como formadora de profesores (Vanegas \& Fuentealba, 2019). Además, las bitácoras me ayudaron a entender, desde otras perspectivas, las relaciones con los profesores en formación, dejando de lado las posturas de autoridad medidas en los conocimientos científicos y dando paso a espacios reflexivos que sirven para la formación de ellos y para mi propio desarrollo profesional. 
Con relación al segundo objetivo específico del self-study, la descripción de convergencias y divergencias entre mi perspectiva y la del investigador, me permitió reflexionar sobre mis procesos reflexivos. Como muestra la figura 3 , este meta-análisis complementa los análisis de las bitácoras y me permite comprender que cuando se coloca la reflexión al centro del acompañamiento de las prácticas pedagógicas, los profesores guías logramos una identización que es robustecida y tensionada por la identidad con el rol y con las formas de relacionarnos con los profesores en formación.

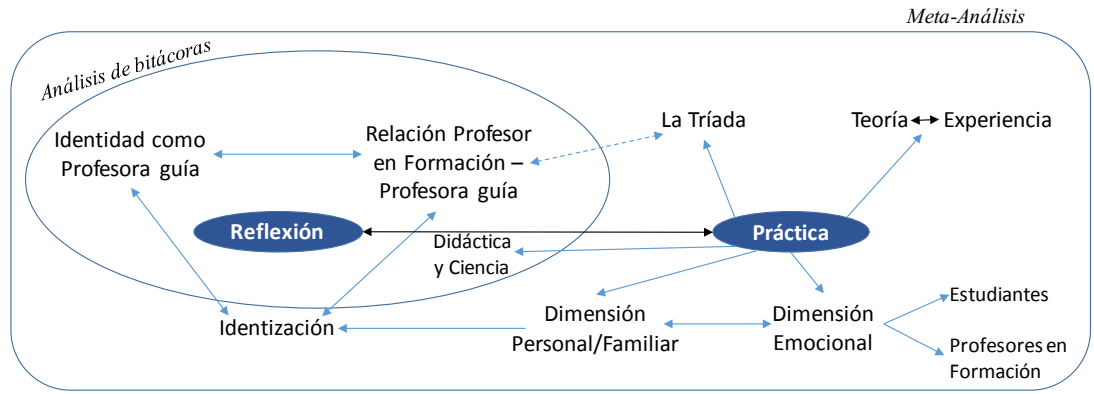

Figura 3. Síntesis de procesos reflexivos y sus relaciones, a partir del self-study. Fuente: elaboración propia.

El meta-análisis me permitió comprender mi propia práctica pedagógica desde una concepción más compleja que cuando es abordada de manera reflexiva, ya que posibilita la incorporación de las perspectivas de la tríada, problematizar la relación entre la teoría y las experiencias de aula. Y más relevante aún, pude darme cuenta sobre el alto nivel de influencia que tienen las dimensiones personal/familiar y emocional en las formas de interacción con los estudiantes y con los profesores en formación, que en definitiva, hacen que se transforme mi identización, o como plantea Korthagen (2010), los propósitos misionales como profesional de la educación.

Hoy, al remirar los datos, considero que no estaba lista para ejercer el rol de profesora formadora de profesores, por existir en mí un sesgo y predisposición frente a los profesores en formación. No contaba con las herramientas formativas para identificarme con el rol de Profesora Guía, como la reflexión y una comprensión más compleja sobre las prácticas pedagógicas; además, no tenía un esquema o protocolo a seguir para realizar esta compleja labor.

Coherente con los planteamientos de Correa (2011), a través de esta investigación me di cuenta de la necesidad imperativa de que como profesora guía debo mantenerme actualizada en el conocimiento teórico, incluso ir más allá de lo que hace un profesor de aula (que no es responsable de formar profesores). Debo estar a la vanguardia no sólo en conocimientos científicos, sino en estrategias pedagógicas y didácticas, debido a que el 
profesor en formación viene con un conocimiento actualizado y en búsqueda de poner en práctica nuevas propuestas de aula. Además, durante estos años de labor como profesora de aula, así como mi rol de profesora guía, me he dado cuenta que la didáctica y la ciencia deben ir de la mano, manteniéndose ambas actualizadas para generar sinergia entre ellas.

También pude identificar que un buen formador de formadores no debe basarse solo en la confianza al encomendar tareas al profesor en formación, sino que debe preparar metodologías y procedimientos para instruir y medir los progresos de éste y así evitar prácticas poco eficaces. Bajo este mismo punto, es obligatorio generar los tiempos y los espacios para reflexionar en conjunto con el profesor en formación, esto implica también que el profesor guía debe tener estos espacios en su programación (Vanegas, 2016).

En el meta-análisis de mis entrevistas, pude identificar una dicotomía entre lo que quiero ser como profesora y la que puedo ser; esta brecha está dada por los obstáculos y dificultades propias del sistema, de los estudiantes y mis propias complejidades como persona y profesional y, de los recursos, medios y tiempo que dispongo para poder analizarlos y generar procesos de mejora e implementarlos.

Analizando los aspectos que me identifican como profesora de aula, pude descubrir que, más allá de querer generar avances en los aprendizajes cognitivos de los estudiantes, lo que pretendo es más bien educar personas, generando en ellos un desarrollo del pensamiento crítico por medio de la entrega de herramientas útiles para la construcción de su futuro.

Mi identificación como profesora guía tiene como enfoque el ser un apoyo en el desarrollo de los procesos reflexivos que permitan a los profesores en formación contextualizar y llevar a la práctica de la mejor manera los conocimientos teóricos aprendidos en el centro de formación; es decir, siguiendo la perspectiva socio-histórica de Kemmis (2009), usar el espacio de práctica pedagógica para, en conjunto con los profesores en formación, poner a prueba los conocimientos teóricos y tensionar la experiencia con la teoría.

En mi rol como profesora de Biología y Ciencias, hoy el desafío es la búsqueda de nuevas estrategias para la enseñanza, que me permitan obtener resultados satisfactorios en instruir en el área de la ciencia, teniendo en cuenta y comprendiendo las diferentes condiciones culturales de los estudiantes. Otro punto importante es la interacción con el estudiantado, la cual debe ser dentro de un marco de cercanía y respeto, permitiendo fortalecer la relación estudiantes-profesor y así obtener un aprendizaje más significativo de los contenidos (conceptuales, procedimentales, actitudinales y socioemocionales). 
Si bien es cierto que debe existir un proceso reflexivo entre el profesor guía y el profesor en formación, es claro que el proceso de la práctica pedagógica no ocurre en un ambiente aislado, es decir, esta reflexión también se puede socializar con otros docentes del establecimiento, no limitando esta socialización a la tríada. Como profesores guías, también podemos hacernos cargo de ayudar a los profesores en formación a conocer e impregnarse de la cultura institucional, de otras responsabilidades profesionales que no ocurren en la sala de clase.

En síntesis, a partir de la experiencia plasmada en este estudio self-study, se abre la posibilidad, y al mismo tiempo muchas interrogantes que nos invitan a seguir trabajando en modelos y procedimientos de acompañamiento que permitan comprender los profesores guías de práctica como objeto de estudio, permitiendo un vínculo profesional más eficaz para la relación tríadica, con el fin de obtener los mejores resultados formativos de la experiencia compartida.

Cabe destacar que esta investigación es un análisis de mi propia experiencia profesional y, si bien sirve como un referente para profundizar en el estudio del rol del profesor guía de prácticas pedagógicas de formación inicial, está limitada metodológicamente, por lo que no admite generalizaciones o extrapolaciones a otros contextos. Por ser un self-study, los resultados de este estudio están condicionados por factores como el carácter de cada participante, las condiciones laborales, el contexto educativo, la identificación como docente y las características propias y únicas de la identización.

El profesor guía, para reconocerse como tal, y comprender las diferencias con su rol como profesor escolar, puede realizar una investigación de su propia práctica como formador de docentes, lo que permite aportar con nuevas comprensiones sobre su desarrollo profesional. Para la reflexión sobre el propio rol como profesor guía, la metodología self-study se convierte en un camino potente que releva los conocimientos y la comprensión de los formadores de formadores en sus propias prácticas, y opera bajo una epistemología que valida los conocimientos y comprensiones generados en la práctica de la formación de profesores (Russell, Fuentealba \& Hirmas, 2016).

A través de esta metodología, los docentes que somos formadores de profesores podremos realizar un examen de la práctica que desarrollamos y así podemos cambiar nuestros valores profesionales y las creencias personales. Esto nos permitiría planificar, implementar y evaluar nuestros procesos de enseñanza y acompañamiento, examinar el impacto real que podemos causar en los aprendizajes de nuestros estudiantes y de los profesores en formación que estemos guiando. Además, la reflexión consciente y sistemática traerá 
como consecuencia un mejor desempeño de la labor profesional y garantizará la adquisición de nuevas perspectivas y herramientas para enfrentar los desafíos para adaptarse a las necesidades de los futuros profesores.

Lo que motiva al formador de formadores a investigar la propia práctica, son los problemas que emergen de su práctica, las cuales desafían a pensar sobre ella de manera diferente. Por ello, Ritter y Bullock (2001 en Hirmas \& Blanco, 2016), enfatizan que se requiere no solo honestidad de su parte, sino también de voluntad de compartir vulnerabilidades e incertidumbres.

\section{Referencias bibliográficas}

Black, P. \& Plowright, D. (2010). A multidimensional model of reflective learning for profesional development. Reflective practice, 11(2), 245-258.

Brewer, J. \& Hunter, A. (2006). Foundations of multimethod research: Synthersizing Styles. Thousand Oaks, CA: Sage.

Clarke, A. \& Erickson, G. (2004). The nature of teaching and learning in self- study. In J. Loughran, L. M, V. LaBoskey \& T. Russell (Eds.), International handbook of Selfstudy of teaching and teacher education practices (Vol. 1, pp. 41- 67). Dordrecht: Kluwer Academic Publishers.

Correa, E. (2011). La práctica docente: una oportunidad de desarrollo profesional. Perspectiva Educacional, 50 (2), 77-95.

Correa, E., Malo, A. \& Gervais, C. (2013). La reflexión compartida, ¿una estrategia de facilitación en inserción profesional? In E. Correa, M. Cividini, R. Fuentealba \& I. Boerr (Eds.), Formación e inserción profesional: desafíos y pistas de facilitación para la profesionalización docente (pp. 137-157). Santiago, Chile: OEI.

Dewey, J. (1933). How we Think: A restatement of the relation of reflective thinking to the educative process. Boston: D.C. Heath and company.

Dewey, J. (1964). Experiencia y Educación. Buenos Aires: Editorial Losada.

Domingo, Á. \& Gómez, V. (2014). La Práctica Reflexiva. Madrid: Narcea.

Figueira, E. M. \& Rivas, M. R. (2011). General tasks of tutors during the Practicum course: reality versus ambition in the development of the tutorial work. Revista de Educacion, 354, 155-181.

Frick, L., Carl, A. \& Beets, P. (2010). Reflection as learning about the self in context: mentoring as catalyst for reflective development in pre-service teachers. South African Journal of Education, 30(3), 421-437.

Gervais, C. (2007). Des enseignants associés explicitent leurs savoirs d'expérience ( $1^{\mathrm{a}}$ ed.). Québec, Canada: Université du Québec.

Hirmas, C. \& Blanco, R. (2016). Introducción. In J. Cornejo, J. Loughran, T. Russell, A. Berry, S. Sandretto, S. Schuck, ... J. J. Mena, Formadores de Formadores, Descubriendo la Propia Voz a través del Self-Study (pp. 7-11). Santiago: BUK Grafica \& Editora. 
Imbernon, F. (2001). Claves para una nueva formación del profesorado. Revista Investigación en la Escuela, 43, 57-66.

Kemmis, S. (2009). Understanding professional practice: a synoptic framework. En B. Green (Ed.), Understanding and Researching Professional Practice (págs. 19-38). Rotterdam, The Netherlands: Sense Publishers.

Korthagen, F. (2010). La práctica, la teoría y la persona en la formación del profesorado. Revista Interuniversitaria de Formación del Profesorado, 24(2), 83-101.

Latorre, M. (2004). Aportes para el análisis de las racionalidades presentes en las prácticas pedagógicas. Estudios Pedagógicos, 30, 75-91.

MINEDUC. (2005). Comisión sobre Formación Inicial Docente. Informe Comisión sobre formación inicial docente. Santiago: MINEDUC.

Montecinos, C. \& Walker, H. (2010). La colaboración entre los centros de práctica y las carreras de pedagogía. Docencia, 42, 64-73.

Olarte, A. C. (2016). El auto estudio como enfoque de investigación en la formación docente. Colombian Applied Linguistics Journal, 18(2), 9-10.

Russell, T., Fuentealba, R. \& Hirmas, C. (2016). Formadores de formadores, descubriendo la propia voz a través del self-study. Santiago: Organización de Estados Iberoamericanos.

Russell, T. \& Mena, J. (2016). El self-study como forma de investigación en la formación del profesorado: Un análisis de contenido de los trabajos presentados en el X Congreso Internacional sobre Self-Study S-STEP de 2014. In J. Cornejo, J. Loughran, T. Russell, A. Berry, S. Sandretto, S. Schuck, . . . Mena, Formadores de Formadores, Descubriendo la Propia Voz a través del Self-Study (p. 236). Santiago: BUK Grafica \& Editora.

Shön, D. (1987). El profesional reflexivo. Cómo piensan los profesionales cuando actúan. España: Ediciones Paidós Ibérica, S.A.

Vallejo, R. \& Finol de Franco, M. (2009). La triangulación como procedimiento de análisis para investigaciones educativas. REDHECS, 7, 117-133.

Vanegas, C. (2016). Procesos Reflexivos de profesores de ciencias durante las prácticas pedagógicas de formación inicial (Tesis Doctoral). Santiago, Chile: Pontificia Universidad Católica de Chile.

Vanegas, C. \& Fuentealba, A. (2019). Identidad profesional docente, reflexión y práctica pedagógica: Consideraciones claves para la formación de profesores. Perspectiva Educacional, 58(1), 115-138. 